\title{
Modeling and Evaluation of Linear Oscillating Actuators
}

\author{
X. Chen* and Z. Q. Zhu*
}

\begin{abstract}
The operation of linear oscillating system is complicated, involving system nonlinearities of both actuator and load, and variations of driving frequency in order to track the mechanical resonance. In this paper, both analytical and state-variable modeling techniques are used to investigate the influence of actuator parameters, such as back-emf/thrust force coefficient and cogging force, on the performance of linear oscillating systems. Analytical derivations are validated by simulations, and good agreements are achieved. The findings of the paper can greatly facilitate the design and evaluation processes of permanent magnet linear actuators.
\end{abstract}

Keywords: Linear actuator, Permanent magnet, State-variable modeling

\section{Introduction}

The operation of linear oscillating system is relatively complicated, usually involving system nonlinearities of both actuator and load, and variations of driving frequency in order to track the mechanical resonance. Hence, the state-variable modeling technique in the time domain is extensively employed to study the dynamic and steady state characteristics of linear oscillating systems. Depending on the linearity of the specific system, such as the load force vs displacement, spring stiffness vs. displacement, actuator thrust force vs. displacement and actuator thrust force vs. supply current etc, either the linear or nonlinear statevariable modeling can be used. For simple analysis and control or cases where no severe nonlinearity is observed, the linear state-variable modeling can be readily adopted, for example in [1] and [2]. However, if nonlinearity is too significant to be neglected, the nonlinear state-variable modeling has to be used. By way of example, both [3] and [4] store the nonlinear finite element (FE) predicted actuator performance in terms of cogging force, force coefficient, inductance, etc as look-up tables, whilst the gas force characteristic of linear compressors can be modeled by an ideal pneumatic model, [5], [6]. Additionally, transient FE analyses can be incorporated into the simulation process, as done in [7] and [8]. Although it is very time-consuming, this is conducive to obtain a more accurate simulation compared to the real behavior of the

\footnotetext{
* Dept. of Electronic and Electrical Engineering, the University of

Sheffield, UK (carnchen@hotmail.com, Z.Q.Zhu@sheffield.ac.uk)

Received 13 October 2012; Accepted 10 November 2012
}

oscillating system.

Despite that there are many publications available discussing the state-variable modeling of linear oscillating systems, very few have studied the inherent influence caused by the nonlinear performance of linear oscillating actuators. Therefore, in this paper the influence of actuator performance, such as back-emf/thrust force coefficient and cogging forces, on oscillating systems are investigated analytically with particular reference to permanent magnet (PM) linear oscillating actuators (LOA). Then, simulations in MATLAB/Simulink are undertaken to validate the foregoing analytical models.

\section{State-variable Modeling of Linear Oscillating Systems}

Generally, the linear oscillating system can be divided into 2 coupled sub-systems, viz. the electromagnetic and mechanical systems. The electromagnetic system converts the electrical energy to mechanical energy via the magnetic fields in the LOA, the developed electromagnetic force being dependant on the supply current and the displacement of moving parts. Neglecting the cross-coupling effect and assuming linear magnetic circuits, the electromagnetic system of a 1-phase PM oscillating system can be modeled by the governing voltage equation formulated as:

$$
u=R i+L \frac{d i}{d t}+i v \frac{d L}{d x}+v \frac{d \psi_{m}}{d x}
$$

where $u$ and $i$ are the voltage and current of power supply; $R$ and $L$ are the winding resistance and self-inductance; $\Psi$ 
and $\Psi_{m}$ are the total winding flux-linkage and the fluxlinkage due to PM; $x$ and $v$ are the displacement and velocity of the moving parts. Based on (1), it can be seen that the reluctance force $\left(F_{R}\right)$ and excitation force $\left(F_{E}\right)$ are given by $(2 \mathrm{a})$ and $(2 \mathrm{~b})$, whilst the total thrust force $\left(F_{a c t}\right)$ produced by LOA is defined as $(2 \mathrm{c})$.

$$
\begin{aligned}
& F_{R}=\frac{1}{2} i^{2} \frac{d L}{d x} \\
& F_{E}=i \frac{d \psi_{m}}{d x}=K_{E} i \\
& F_{a c t}=F_{C}+F_{R}+F_{E}
\end{aligned}
$$

where $K_{E}$, namely the back-emf/force coefficient, is the derivative of the winding flux-linkage due to PM over the displacement, while the equation of cogging force $\left(F_{C}\right)$ not given due to its complex nature.

Secondly, the mechanical state-variable equation, (3), can be employed to predict the mechanical behavior of linear oscillating systems.

$$
F_{a c t}-F_{L}-K_{s} x-D_{e} v=m_{e} \frac{d^{2} x}{d t^{2}}
$$

where $F_{L}$ is the load force; $m_{e}$ is the effective moving mass; $K_{s}$ is the effective stiffness of the mechanical spring; $D_{e}$ is the effective viscous damping coefficient of the oscillating system mainly due to the hysteresis loss of the spring and the bearing friction. As for the load force, there are several different fixtures available, typically the frictional load, linear generator load, linear compressor load, etc. However, the fundamental approach for the modeling of different load forces is identical, i.e. to convert the load forces into equivalent stiffness and damping components and substitute it with them in (3).

It should be noted that although it is clearly the most straightforward and cost-effective way to drive linear oscillating systems by sinusoidal or rectangular voltage supplies, this is incapable of fully characterizing the system because it ultimately relies on the induced current to determine the actuator performance. Whereas the current control method is equally applicable to linear oscillating systems, just as in rotary machines. Under the sinusoidal current control, the benefit of higher efficiency is observed in [9], whilst [6] finds that the instable jump problem of the oscillating amplitude, [10], is effectively eliminated. Therefore, the current control method will be used in this paper by commanding the supply current to be:

$$
i=I_{0} \sin (\omega t)
$$

\section{Influence of Actuator Parameters on}

\section{Performance of Oscillating Systems}

Normally, in order to simplify the analysis only the excitation force with $K_{E}$ being constant irrespective of its variation versus displacements is considered, whilst the cogging force and reluctance force are both treated as zero. However, in the real working condition, all of them will introduce nonlinearities to the linear oscillating system and ultimately affect the system performance. Therefore, the influence of $F_{E}, F_{C}$ and $F_{R}$ on the oscillating system is investigated in this section.

\subsection{Excitation Force}

In some topologies of LOAs, e.g. SPM with small slot openings, the cogging force $F_{C}$ can be negligible, whilst the reluctance force $F_{R}$ can be regarded as zero due to negligible change of winding self-inductance. Hence, the thrust force produced by LOA is only comprised of $F_{E}$. As can be seen from $(2 \mathrm{~b}), F_{E}$ is largely dependent on the backemf/force coefficient $K_{E}$, which is also a function of the displacement $x$. Therefore, the influence of $F_{E}$ on the oscillation can be analyzed with regard to different $K_{E^{-}} x$ characteristics.

\section{A. $K_{E}=$ Constant}

If $K_{E}$ remains constant irrespective of the variation of displacements, the mechanical state-variable equation is:

$$
K_{E} I_{0} \sin (\omega t)-K_{s} x-D_{e}^{\prime} v=m_{e} \frac{d^{2} x}{d t^{2}}
$$

where for simplification the pure damping load $\left(F_{L}=D_{e l} v\right)$ is considered; $D_{e l}$ and $D_{e}^{\prime}$ are the load and total equivalent viscous damping coefficients.

Due to the low-pass filter effect of the mechanical massspring-damper system, the steady-state displacement is essentially sinusoidal as:

$$
x=A \sin (\omega t-\alpha)
$$

where the oscillating amplitude $A$ and the phase angle $\alpha$ between $i$ and $x$ are:

$$
A=\frac{K_{E} I_{0}}{\sqrt{\left(K_{s}-m_{e} \omega^{2}\right)^{2}+\left(D_{e}^{\prime} \omega\right)^{2}}} \quad \alpha=\tan ^{-1} \frac{D_{e}^{\prime} \omega}{K_{s}-m_{e} \omega^{2}}
$$

whilst based on (5) and multiply it by the velocity gives: 


$$
P_{e m}=\frac{1}{2} D_{e} \omega^{2} A^{2}+\frac{1}{2} D_{e l} \omega^{2} A^{2}=P_{d l}+P_{o}
$$

where $P_{d l}, P_{e m}$ and $P_{o}$ are the system internal damping loss, electromagnetic and output powers. Neglecting the actuator iron loss, the system efficiency $\eta_{s y s}$ is given by:

$$
\eta_{s y s}=\frac{D_{e l} \omega^{2} A^{2}}{R I_{0}{ }^{2}+D_{e}^{\prime} \omega^{2} A^{2}}=\frac{D_{e l}}{\frac{R}{K_{E}^{2}}\left[\left(\frac{K_{s}}{\omega}-m_{e} \omega\right)^{2}+D_{e}^{\prime 2}\right]+D_{e}^{\prime}}
$$

It is evident that $\eta_{s y s}$ achieves maximum (mechanical resonance) when:

$$
\left.\omega_{n}=\sqrt{\frac{K_{s}}{m_{e}}} \quad x_{n}=-\frac{K_{E} I_{0}}{D_{e}^{\prime} \omega_{n}} \cos \omega_{n} t\right) \quad P_{e m \cdot n}=\frac{K_{E}^{2} I_{0}^{2}}{2 D_{e}^{\prime}}
$$

\section{B. $K_{E}$ being a linear function of displacement}

If the $K_{E}(x)$ characteristic exhibits profiles as shown in Fig. 1, the mechanical equation is:

$$
K_{E} x I_{0} \sin (\omega t)-K_{s} x-D_{e}^{\prime} v=m_{e} \frac{d^{2} x}{d t^{2}}
$$

Assuming the steady-state solution having the same formation as (6) and neglecting harmonics, (9) becomes:

$$
A\left(K_{s}-m_{e} \omega^{2}\right) \sin (\omega t-\alpha)+D_{e}^{\prime} A \omega \cos (\omega t-\alpha)=\frac{1}{2} K_{E} A I_{0} \cos (\alpha)
$$

It is can be seen that $A$ has to be zero so that (10) can be satisfied, meaning this case of $K_{E}$ is incapable of producing stable sinusoidal oscillation.

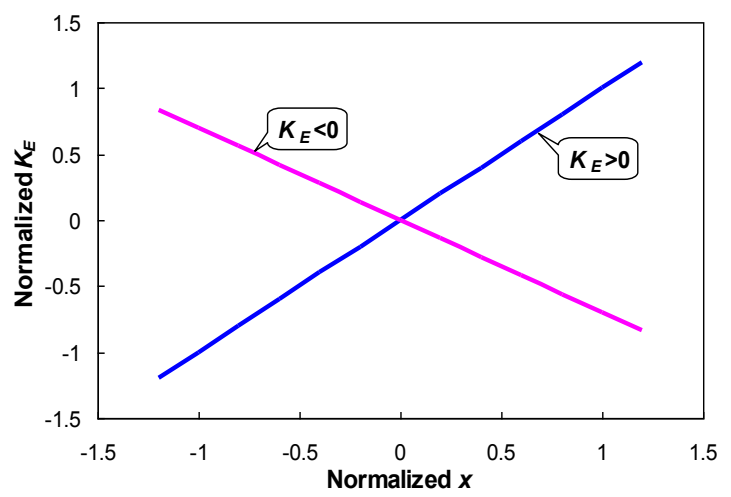

Fig. 1. Linear-slope $K_{E}(x)$ characteristics

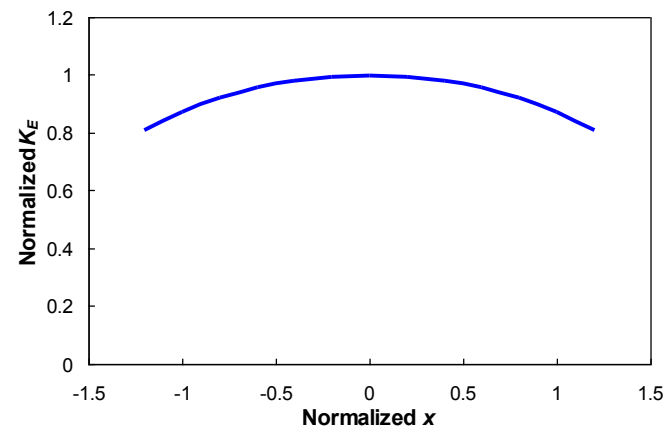

Fig. 2. Nonlinear $K_{E}(x)$ characteristics

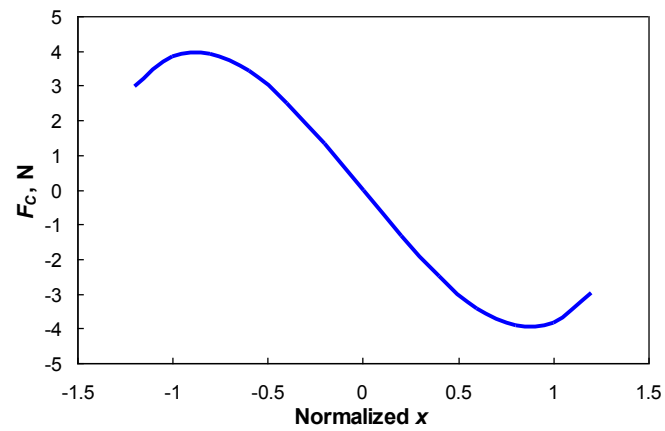

Fig. 3. An example of nonlinear $F_{C}(x)$ characteristic

C. Nonlinear $K_{E}$

As can be seen from Case $B$, in order to produce oscillation the $K_{E^{-}} x$ curve must not cross zero. However, the "roll-off" near the end of the rated stroke may exist in the $K_{E^{-}} x$ characteristic, making it look like the profile shown in Fig. 2. In this case, the characteristic can be curve-fitted over the rated stroke, and the $K_{E}$ can be approximated as:

$$
K_{E}(x)=K_{E 0}-K_{E 2} x^{2}-K_{E 4} x^{4}-K_{E 6} x^{6} \cdots
$$

where $A_{R}$ is the rated stroke and $-A_{R} \leq x \leq A_{R}$.

For simplicity $K_{E}(x)=K_{E 0}-K_{E 2} x^{2}\left(K_{E 0}, K_{E 2}>0\right)$ is used for analysis, and the mechanical equation becomes:

$$
\left(K_{E 0}-K_{E 2} x^{2}\right) I_{0} \sin (\omega t)-K_{s} x-D_{e}^{\prime} v=m_{e} \frac{d^{2} x}{d t^{2}}
$$

Assuming the steady-state solution having the same formation as (6) and neglecting harmonics, (12) becomes: $A\left(K_{s}-\omega^{2} m_{e}\right) \sin (\omega t-\alpha)+A \omega D_{e}^{\prime} \cos (\omega t-\alpha)=$ $\left(K_{E 0} I_{0}-\frac{3 K_{E 2} I_{0} A^{2}}{4}\right) \cos (\alpha) \sin (\omega t-\alpha)+\left(K_{E 0} I_{0}-\frac{K_{E 2} I_{0} A^{2}}{4}\right) \sin (\alpha) \cos (\omega t-\alpha)$

Comparing the left and right sides of (13), $A$ and $\alpha$ can be 
obtained by solving equation (14).

$$
\left\{\begin{array}{c}
A\left(K_{s}-\omega^{2} m_{e}\right)=\left(K_{E 0} I_{0}-\frac{3 K_{E 2} I_{0} A^{2}}{4}\right) \cos (\alpha) \\
A \omega D_{e}^{\prime}=\left(K_{E 0} I_{0}-\frac{K_{E 2} I_{0} A^{2}}{4}\right) \sin (\alpha)
\end{array}\right.
$$

Due to the complication of (14), it is very difficult to derive a simple equation to determine the true resonant frequency. Alternatively, a quasi-resonant frequency can be regarded as:

$$
\omega_{q n}=\sqrt{K_{s} / m_{e}}
$$

while the mover displacement and electromagnetic power at the quasi-resonant condition are:

$$
\begin{gathered}
x=-A_{q n} \cos \left(\omega_{q n} t\right) \\
P_{e m \cdot q n}=\frac{1}{2} D_{e}^{\prime} \omega_{q n}^{2} A_{q n}^{2}=\frac{1}{2 D_{e}^{\prime}}\left(K_{E 0} I_{0}-\frac{K_{E 2} I_{0} A_{q n}^{2}}{4}\right)^{2} \\
\text { where } A_{q n}=2 \frac{\sqrt{\omega_{q n}^{2} D_{e}^{\prime 2}+K_{E 0} K_{E 2} I_{0}^{2}}-\omega_{q n} D_{e}^{\prime}}{K_{E 2} I_{0}} .
\end{gathered}
$$

It can be seen that compared to Case $A$ with constant $K_{E}$, the nonlinear $K_{E}$ has an adverse influence on the performance of the linear oscillating system, such as the reduction of oscillating amplitude, electromagnetic power and efficiency.

\subsection{Cogging Force}

In some LOA topologies with significant cogging force, the influence of cogging force on the oscillation has to be considered. In this part, its influence is investigated according to its distinctive gradient versus displacement, while the reluctance force $F_{R}$ and the load force $F_{L}$ are 0 and $D_{e l} v$, respectively.

$$
\text { A. } F_{C}=k_{c} \cdot x
$$

If the $F_{C^{-}} x$ profile is represented by an ideal linear curve, the mechanical equation can be simplified as:

$$
K_{E} I_{0} \sin (\omega t)-\left(K_{s}-k_{c}\right) x-D_{e}^{\prime} v=m_{e} \frac{d^{2} x}{d t^{2}}
$$

As can be seen, when $k_{c}>0$ the cogging force offsets the spring stiffness $K_{s}$, which is not recommended as the stiffness of the actual spring has to be increased in order to maintain the resonant operation at the supply frequency. However, if $k_{c}<0$ the cogging force enhances the spring stiffness, which, in other words, is conducive to reduce the stiffness of the actual spring to maintain the resonant oscillation at the supply frequency.

\section{B. Nonlinear $F_{C}$}

As can be seen from Case $A$, the $F_{C^{-}} x$ characteristic with negative gradient is more beneficial to the oscillation. However, the "roll-off" may be very common in the $F_{C^{-}} x$ characteristic, Fig. 3. In this regard, the characteristic can be curve-fitted over the effective stroke range as:

$$
F_{C}(x)=-k_{c 1} x+k_{c 3} x^{3}+k_{c 5} x^{5}+k_{c 7} x^{7} \cdots
$$

where $k_{c l}>0$ and $-A_{R} \leq x \leq A_{R}$.

In order to simplify the analysis, $F_{C}(x)=-k_{c l}+k_{c 3} x^{3}\left(k_{c l}\right.$, $\left.k_{c 3}>0\right)$ is used, and the mechanical equation can be rewritten as:

$$
K_{E} I_{0} \sin (\omega t)-K_{s}^{\prime} x+k_{c 3} x^{3}-D_{e}^{\prime} v=m_{e} \frac{d^{2} x}{d t^{2}}
$$

where $K_{s}^{\prime}=K_{s}+k_{c l}$.

The steady-state solution to (17) can be assumed as (6), while neglecting other harmonics (17) becomes:

$$
A\left(K_{s}^{\prime}-m_{e} \omega^{2}-\frac{3 k_{c 3} A^{2}}{4}\right) \sin (\omega t-\alpha)+D_{e}^{\prime} \omega A \cos (\omega t-\alpha)=K_{E} I_{0} \sin (\omega t)
$$

Comparing the left and right sides, $A$ and $\alpha$ can be obtained by solving (19):

The electromagnetic power is give by:

$$
P_{e m}=\frac{D_{e}^{\prime}}{2} \frac{K_{E}^{2} I_{0}^{2}}{\left(\frac{\left.K_{s}^{\prime}-m_{e} \omega^{2}-\frac{3 k_{c 3} A^{2}}{4}\right)^{2}}{\omega}+D_{e}^{\prime 2}\right.}=\frac{1}{2} D_{e}^{\prime} \omega^{2} A^{2}
$$

As can be seen, $P_{e m}$ will reach maximum when:

$$
K_{s}^{\prime}-m_{e} \omega^{2}-\frac{3 k_{c 3} A^{2}}{4}=0
$$

Thus, the resonant frequency $\omega_{n}$ and oscillating amplitude $A_{n}$ are given by:

$$
\left\{\begin{array}{c}
D_{e}^{\prime} \omega_{n} A_{n}=K_{E} I_{0} \\
K_{s}^{\prime}-m_{e} \omega_{n}^{2}-\frac{3 k_{c 3} A_{n}^{2}}{4}=0
\end{array}\right.
$$

while $\alpha_{n}$ is $90^{\circ}$, the mover displacement and electromagnetic power are:

$$
\begin{gathered}
x=-A_{n} \cos \left(\omega_{n} t\right) \\
P_{e m \cdot n}=\frac{1}{2} D_{e}^{\prime} \omega_{n}^{2} A_{n}^{2}=\frac{K_{E}^{2} I_{0}^{2}}{2 D_{e}^{\prime}}
\end{gathered}
$$


In summary, this case with nonlinear $F_{C}$ affects the resonant frequency and oscillating amplitude, whereas the phase angle between $i$ and $x$, maximum electromagnetic power at resonance remain unchanged compared to that without $F_{C}$.

Furthermore, if the $F_{C^{-}} x$ characteristic is:

$$
F_{C}\left(A_{R}\right)=-k_{c 1} A_{R}+k_{c 3} A_{R}^{3} \leq 0
$$

and $A_{n} \leq A_{R}$, (21) becomes:

$$
\begin{aligned}
& K_{s}^{\prime}-m_{e} \omega_{n}^{2}-\frac{3 k_{c 3} A_{n}^{2}}{4} \geq K_{s}^{\prime}-m_{e} \omega_{n}^{2}-\frac{3 k_{c 3} A_{R}^{2}}{4} \\
& \Rightarrow K_{s}^{\prime}-m_{e} \omega_{n}^{2}-\frac{3 k_{c 3} A_{n}^{2}}{4} \geq K_{s}+\frac{k_{c 1}}{4}-m_{e} \omega_{n}^{2}
\end{aligned}
$$

Therefore, $\omega_{n}$ at resonance is:

$$
\sqrt{\frac{K_{s}+\frac{k_{c 1}}{4}}{m_{e}}} \leq \omega_{n}<\sqrt{\frac{K_{s}^{\prime}}{m_{e}}}
$$

It can be seen that the cogging force with nonlinear $F_{C^{-}} x$ characteristic is still capable of enhancing the effective spring stiffness, although its contribution can be significantly compromised by $k_{c 3}$ compared to Case $A$ with $k_{c}<0$.

\section{Simulations}

A tubular moving-magnet actuator based on [5], the schematic being shown in Fig.4, is utilized in this section as the test LOA for both state-variable modeling and numerical simulation.

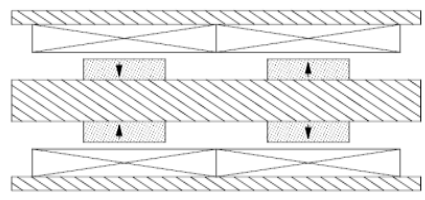

Fig.4. Schematic of a tubular moving-magnet actuator

Table 1. Main parameters of the actuator in Fig. 4

\begin{tabular}{|c|c|c|c|}
\hline$R, \Omega$ & 7 & $L, \mathrm{mH}$ & 5.0 \\
\hline$K_{E}, \mathrm{~N} / \mathrm{A}$ & 4.2 & $m_{e}, \mathrm{~kg}$ & 0.039 \\
\hline$K_{s}, \mathrm{~N} / \mathrm{m}$ & 1884 & $D_{e}, \mathrm{~s} / \mathrm{m}$ & 3 \\
\hline$A_{R}, \mathrm{~mm}$ & 4 & & \\
\hline
\end{tabular}

\subsection{Back-emf/force coefficient}

Firstly, if the nonlinearity of back-emf/force coefficient is ignored, the main parameters of the actuator can be summarized in Table 2.1. Using Simulink, the LOA model is implemented as shown in Fig. 5, in which the current source is pre-set with fixed magnitude of $0.6 \mathrm{~A}$ and variable frequency. Fig. 6 shows the analytically calculated and simulated results of $A, \alpha, P_{e m}$ and $\eta$ on the test LOA, indicating the resonant frequency being $\sim 35 \mathrm{~Hz}$ and the maximum efficiency being $\sim 45 \%$.

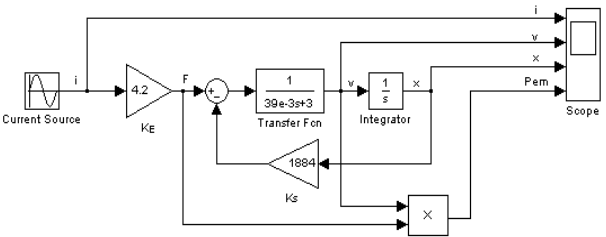

Fig. 5. Simulink block diagram for LOA system

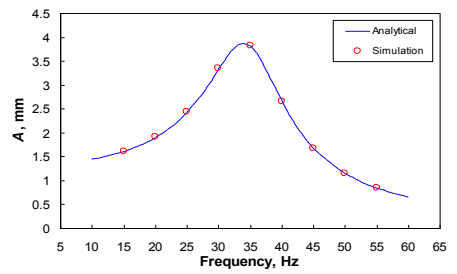

(a) Amplitude of oscillation

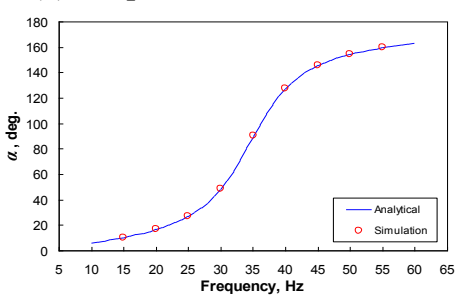

(b) $i-x$ phase shift angle

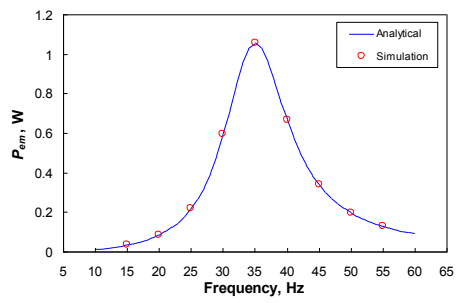

(c) Electromagnetic power

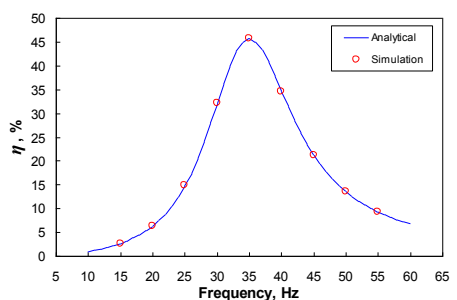

(d) Efficiency

Fig. 6. Comparison of analytical and simulated results

Secondly, to account for the nonlinearity of backemf/force coefficient, the test LOA is used for the comparison of analytical and simulation results, whose main parameters are listed in Table 2. Fig. 7 shows the LOA model implemented in Simulink, in which the current source is pre-set with fixed magnitude of $0.6 \mathrm{~A}$ but variable frequency, whilst Fig. 8 compares the analytically predicted 
and simulated results of $A, P_{e m}$ and $\eta$ on the test LOA, indicating the resonant frequency still remaining at $\sim 35 \mathrm{~Hz}$ and the maximum efficiency being dropped to $\sim 43.5 \%$ compared to the previous $\sim 45 \%$ in the previous case, Fig. 9 .

Table 2. Main parameters of the test actuator

\begin{tabular}{|c|c|c|c|}
\hline$R, \Omega$ & 7 & $L, \mathrm{mH}$ & 5.0 \\
\hline$K_{E 0}, \mathrm{~N} / \mathrm{A}$ & 4.2 & $K_{E 2}, \mathrm{~N} /\left(\mathrm{Am}_{2}\right)$ & 52500 \\
\hline$K_{s}, \mathrm{~N} / \mathrm{m}$ & 1884 & $m_{e}, \mathrm{~kg}$ & 0.039 \\
\hline$A_{R}, \mathrm{~mm}$ & 4 & $D_{e}, \mathrm{~s} / \mathrm{m}$ & 3 \\
\hline
\end{tabular}

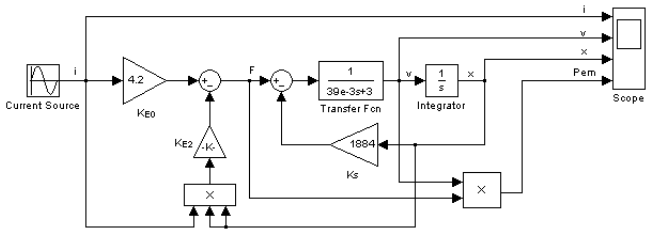

Fig. 7. Simulink block diagram for LOA system

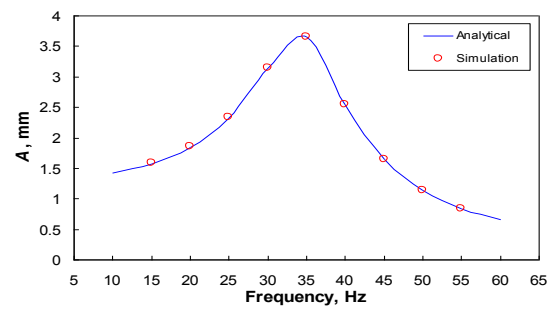

(a) Amplitude of oscillation

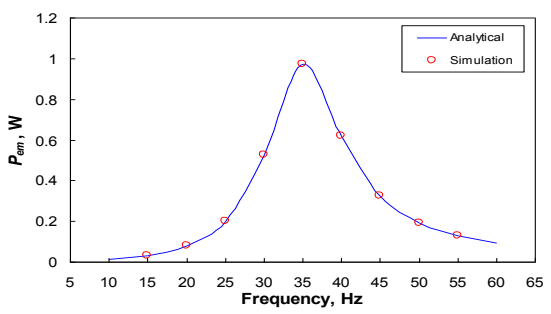

(b) Electromagnetic power

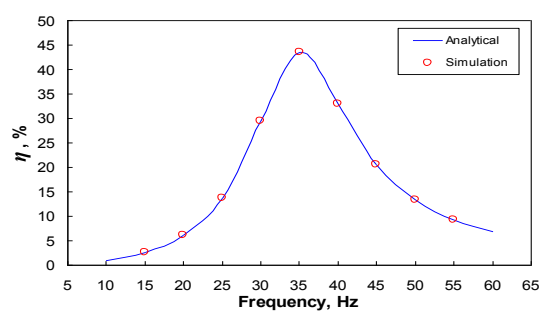

(c) Efficiency

Fig. 8. Comparison of analytical and simulated results

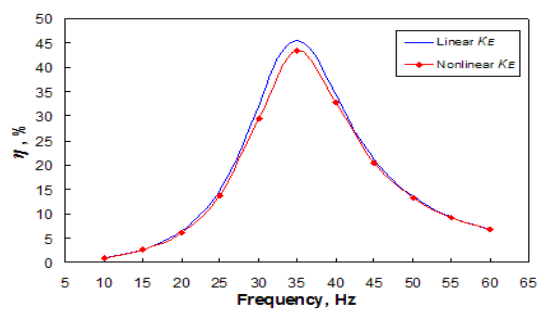

Fig. 9. Efficiency comparison of constant and nonlinear $K_{E}$

\subsection{Cogging force}

Table 3. Main parameters of the test actuator

\begin{tabular}{|c|c|c|c|}
\hline$R, \Omega$ & 7 & $L, \mathrm{mH}$ & 5.0 \\
\hline$K_{E}, \mathrm{~N} / \mathrm{A}$ & 4.2 & $k_{c}, \mathrm{~N} / \mathrm{m}$ & 1750 \\
\hline$A_{R}, \mathrm{~mm}$ & 4 & $m_{e}, \mathrm{~kg}$ & 0.039 \\
\hline$K_{s}, \mathrm{~N} / \mathrm{m}$ & 1884 & $D_{e}, \mathrm{Ns} / \mathrm{m}$ & 3 \\
\hline
\end{tabular}

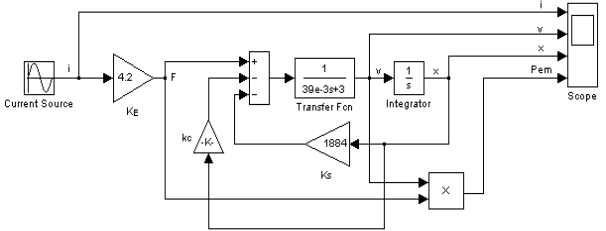

Fig. 10. Simulink block diagram for LOA system

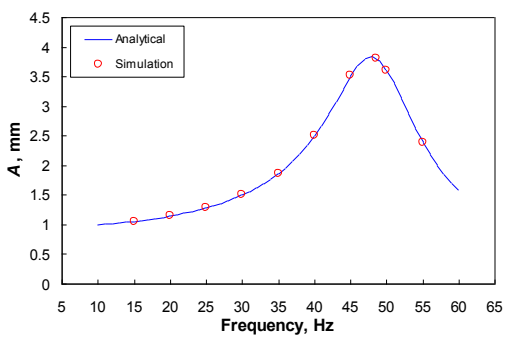

(a) Amplitude of oscillation

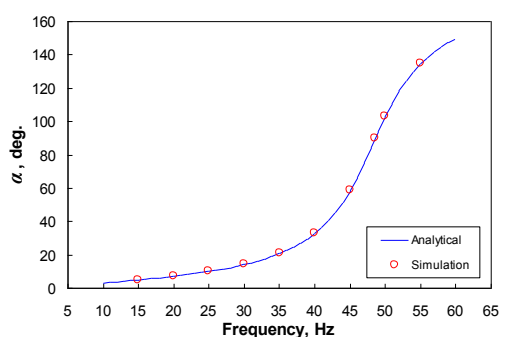

(b) $i-x$ phase shift angle

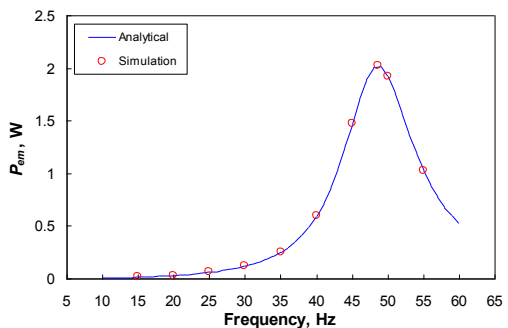

(c) Electromagnetic power

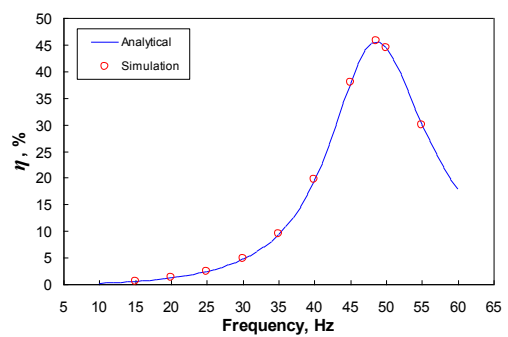

(d) Efficiency

Fig. 11. Comparison of analytical and simulated results 
Firstly, the comparison of analytical and simulation results are undertaken based on the Simulink model shown in Fig. 10, in which the current source is pre-set with fixed magnitude of 0.83 A but variable frequency, while other major parameters are listed in Table 3. Fig. 11 is the comparisons of the analytically predicted and simulated results of $A, \alpha, P_{e m}$ and $\eta$ on the test LOA, showing the resonant frequency being shifted to $\sim 48.6 \mathrm{~Hz}$ and the maximum efficiency remaining at $\sim 45.6 \%$ the same as in Fig. 6.

Secondly, if nonlinear cogging force is considered, the simulation is undertaken based on the Simulink model shown in Fig. 12, in which the current source is pre-set with fixed magnitude of 0.83 A but with variable frequency, while other major parameters can be referred to as in Table 4. Fig. 13 compares the analytically predicted and simulated results of $A, \alpha, P_{e m}$ and $\eta$ on the test LOA. As can be seen, relatively good agreements are achieved between analytical and simulated results, while the discontinuity of responses, often referred to as the jump phenomenon, near resonance is observed in simulations. The predicted resonant frequency by analytical analyses is $\sim 50.03 \mathrm{~Hz}$ with $P_{e m}$ and $\eta$ being $\sim 2.025 \mathrm{~W}$ and $\sim 45.65 \%$, respectively, whilst the resonant frequency by simulation is identified as $\sim 50.6 \mathrm{~Hz}$ (relative difference $<1.3 \%$ ), but with essentially the same maximum $P_{e m}$ and $\eta$ (being $\sim 2.019 \mathrm{~W}$ and $\sim 45.58 \%$ respectively) as the analytical data.

Table 4. Main parameters of the test actuator

\begin{tabular}{|c|c|c|c|}
\hline$R, \Omega$ & 7 & $L, \mathrm{mH}$ & 5.0 \\
\hline$K_{E}, \mathrm{~N} / \mathrm{A}$ & 4.2 & $A_{R}, \mathrm{~mm}$ & 4 \\
\hline$k_{c l}, \mathrm{~N} / \mathrm{m}$ & 3859 & $k_{c 3}, \mathrm{~N} / \mathrm{m}^{3}$ & $1.843 \times 10^{8}$ \\
\hline$m_{e}, \mathrm{~kg}$ & 0.039 & $D_{e}, \mathrm{Ns} / \mathrm{m}$ & 3 \\
\hline$K_{s}, \mathrm{~N} / \mathrm{m}$ & 1884 & & \\
\hline
\end{tabular}

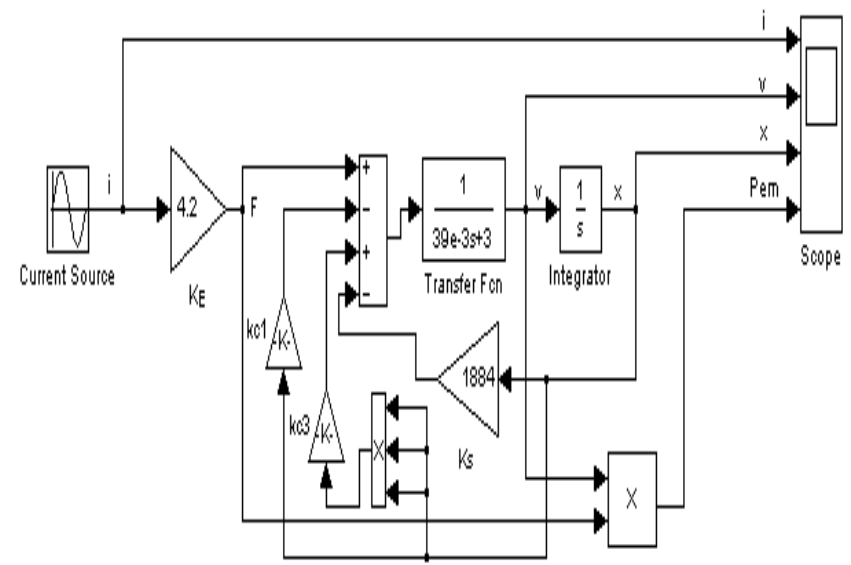

Fig. 12. Simulink block diagram for LOA system

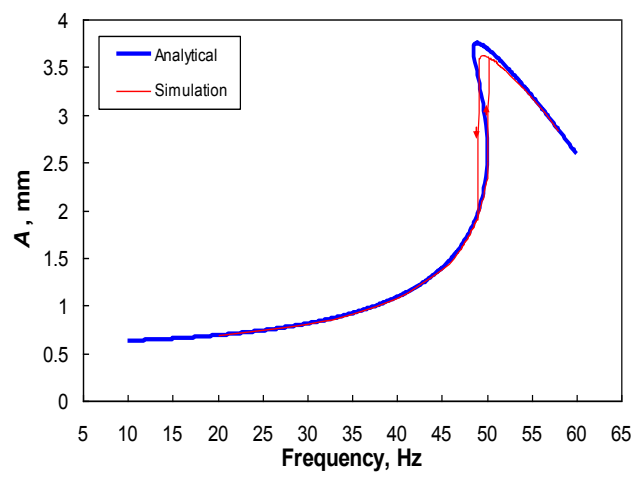

(a) Amplitude of oscillat ion

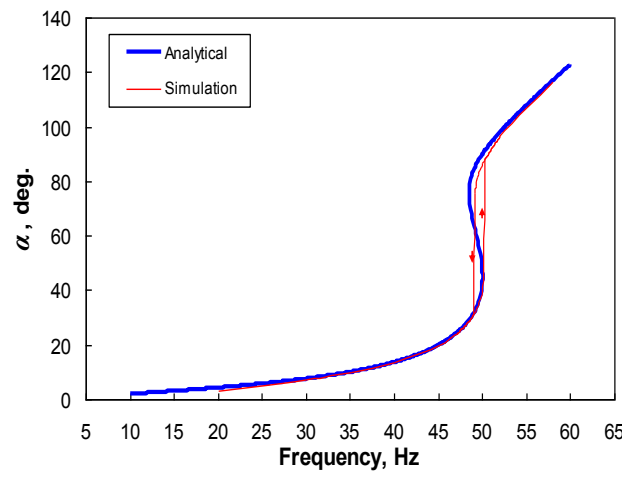

(b) $i-x$ phase shift angle

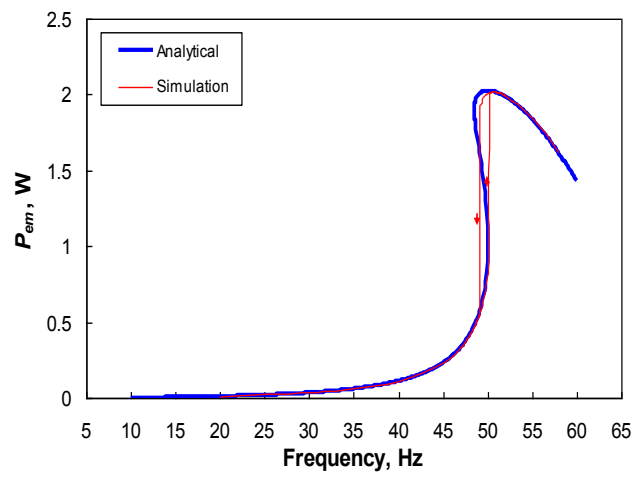

(c) Electromagnetic power

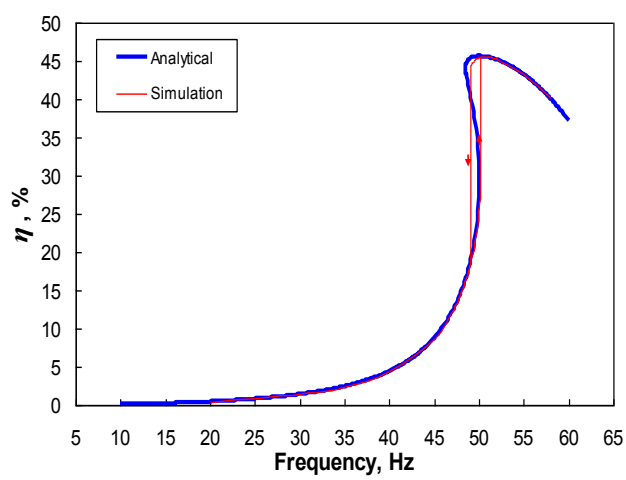

(d) Efficiency

Fig. 13. Comparison of analytical and simulated results 


\section{Conclusion}

In this paper, both analytical and state-variable modeling techniques are used to investigate the influence of actuator parameters, such as back-emf/thrust force coefficient, cogging forces, on the performance of oscillating systems. Then the analytical derivations are validated by simulations, and good agreements are achieved. The conclusions of the paper potentially are capable of facilitating the design and evaluation of various PM linear actuators.

\section{References}

[1] T. W. Chun, J. R. Ahn, J. Y. Yoo and C. W. Lee, "Analysis and Control for Linear Compressor System Driven by PWM Inverter," Proc. of the 30th annual conference of IEEE Industrial Electronics Society, pp. 263-267, Nov. 2004.

[2] S.M. Jang, J.Y. Choi, D.J. You, H.W. Cho, "The Influence of Mechanical Spring on the Dynamic Performance of a Moving-magnet Linear Actuator with Cylindrical Halbach Array," in Proceedings of the 40th IEEE Industry Applications Society Annual Meeting (IAS'2005), pp. 21322139, Oct. 2005, Hong Kong, China.

[3] S. A. Evans, I.R. Smith, J.G. Kettleborough, "Permanentmagnet Linear Actuator for Static and Reciprocating Shortstroke Electromechanical Systems," IEEE/ASME Transactions on Mechtronics, vol. 6, no. 1, pp. 36-42, Mar. 2001.

[4] L. N. Tutelea, M. C. Kim, M. Topor, et al, "Linear Permanent Magnet Oscillatory Machine: Comprehensive Modeling for Transients with Validation by Experiments," IEEE Transactions on Industrial Electronics, vol. 55, no. 2, pp. $492-$ 500, 2008.

[5] R.E. Clark, "Resonant Reciprocating Actuation Systems for Air-compressors," Ph.D. Thesis, University of Sheffield, Jul. 1999.

[6] J. Wang, Z. Lin and D. Howe, "Characteristics of Linear Compressors under Current Source Excitation," Proc. IMechE Part A: J. Power and Energy, vol. 221, pp. 10571065, 2007.

[7] R. E. Clark, G. W. Jewell and D. Howe, "Dynamic Modeling of Tubular Moving-magnet Linear Actuators," Journal of Applied Physics, vol. 93, no. 19, pp. 8787-8789, 2003.

[8] B. Tomczuk, M. Sobol, "A Field-network Model of a Linear Oscillating Motor and its Dynamic Characteristics," IEEE Transactions on Magnetics, vol. 41, no. 8, pp. 2362-2367, 2005.
[9] M. Utsuno, M. Takai, T. Mizuno, H. Yamada, "Comparison of the Losses of a moving-magnet Type Linear Oscillatory Actuator under Two Driving Methods," IEEE Transactions on Magnetics, vol. 38, no. 5, pp. 3300-3303, Sep. 2002.

[10] G. S. Choe and K. J. Kim, "Analysis of Non-linear Dynamics in Linear Compressor," JSME International Journal Series C, vol. 43 , no. 3 , pp. 545-552, 2000.

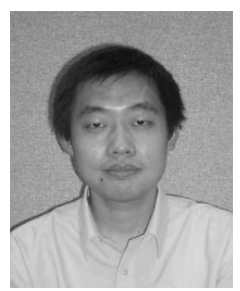

X. Chen received the B.Eng. and M.Eng. degrees from the College of Electrical Engineering, Zhejiang University, Hangzhou, China, in 2001 and 2004, respectively, and the $\mathrm{PhD}$ degree from the Department of Electronic and Electrical Engineering,

University of Sheffield, UK, in 2012.

He is now working at Dyson, UK. His research interest is linear and rotary permanent magnet machines and control.

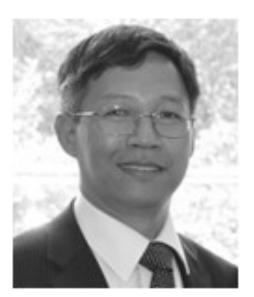

Z. Q. Zhu received the B.Eng. and M.Sc. degrees in electrical and electronic engineering from Zhejiang University, Hangzhou, China, in 1982 and 1984, respectively, and the Ph.D. degree in electrical and electronic engineering from the University of Sheffield, Sheffield, U.K., in 1991.

Since 1988, he has been with the University of Sheffield, where he is currently a Professor at the Department of Electronic and Electrical Engineering, Head of the Electrical Machines and Drives Research Group, Academic Director of Sheffield Siemens Wind Power Research Centre. His current major research interests include design and control of permanent magnet brushless machines and drives, for applications ranging from automotive to renewable energy. He is a Fellow of IEEE. 